\title{
Global particle-in-cell simulations of plasma pressure effects on Alfvénic modes
}

\author{
Alexey Mishchenko* \\ Max-Planck-Institut für Plasmaphysik, \\ EURATOM-Association, D-17491 Greifswald, Germany \\ Axel Könies \\ Max-Planck-Institut für Plasmaphysik, \\ EURATOM-Association, D-17491 Greifswald, Germany \\ Roman Hatzky \\ High Level Support Team (HLST), Max-Planck-Institut für Plasmaphysik, \\ EURATOM-Association, D-85748 Garching, Germany
}

(Dated: January 3, 2011)

\begin{abstract}
Global linear gyrokinetic particle-in-cell (PIC) simulations of electromagnetic modes in realistic tokamak geometry are reported. The effect of plasma pressure on Alfvénic modes is studied. It is shown that the fast-particle pressure can considerably affect the shear Alfvén wave continuum structure and hence the toroidicity-induced gap in the continuum. It is also found that the energetic ions can substantially reduce the growth rate of the ballooning modes (and perhaps completely stabilize them in a certain parameter range). Ballooning modes are found to be the dominant instabilities if the bulk plasma pressure gradient is large enough.
\end{abstract}

* alexey.mishchenko@ipp.mpg.de 


\section{INTRODUCTION}

Fast particles can destabilize various shear Alfvén waves (SAW) [1] (normally through the gradient of the fast-particle density). The associated transport of the fast particles [2] can result in reduced heating efficiency thus impacting the device performance. Another consequence is an increased heat load on the plasma wall (hot spots) and even damage of the wall [3]. One of the most important SAW modes in tokamak plasmas is the Toroidal Alfvén Eigenmode (TAE) [4-6]. Destabilization of the TAE modes occurs if the mode drive resulting from interaction of a shear Alfvén wave with fast particles exceeds the mode damping. In Refs. [7, 8], it has been demonstrated that fast-particle Finite Larmor Radius (FLR) and Finite Orbit Width (FOW) effects can play a strongly stabilizing role. Landau damping on thermal ions [9] is another possible mechanism. Continuum damping [10-12] and radiative damping [13-15] are both associated with the interaction of TAE modes with the SAW continuum (caused by the bulk-plasma FLR effects). The structure of the SAW continuum is determined by the bulk plasma density and safety factor profiles (Alfvén velocity) but also by the coupling between the SAW and acoustic branches [16]. This coupling is proportional to the plasma pressure and thus depends both on the bulk plasma temperature and density profiles (in the following, we will see that the fast-particle pressure must be taken into account as well). The damping due to collisions between trapped and passing electrons $[9,17]$ depends on the electron density and temperature profiles, too.

One can see that most of the damping mechanisms are associated with the properties of the background plasma. On the other hand, the free energy stored in the thermal ions can destabilze certain modes, especially in the low-frequency range of the SAW spectrum [18]. In order to quantitatively assess all these effects, a kinetic description is desirable.

The first-principle gyrokinetic approach has been employed in Refs. [19, 20] (global eigenvalue code LIGKA) to study the continuum and radiative damping mechanisms. The electron-fluid ion-kinetic hybrid gyrokinetic model has been benchmarked vs. the theoretical predictions for the TAE mode in Refs. [21, 22] (global particle-in-cell code GTC) and Ref. [23] (global particle-in-cell code GEM). Local flux-tube gyrokinetic simulations have also been performed with the Eulerian gyrokinetic code GYRO.

In Refs. [8, 24], the global first-principle gyrokinetic particle-in-cell (PIC) code GYGLES was used to study TAE modes and their destabilization by fast particles. A stabilizing role 
of fast-particle FLR and FOW effects was demonstrated. Also, an energetic particle mode was found in the case where the fast-particle drive exceeded some threshold value. In the gyrokinetic PIC approach, all the effects associated with the global non-perturbative nonideal character of the Alfvénic instabilities in fusion plasmas are automatically included. Also, the effects associated with the finite extent of the particle orbits are treated naturally. The cancellation problem $[25,26]$ associated with the canonical-momentum formulation of Ampere's law is solved employing the control-variate method described in Ref. [27].

In the present paper, we use the global gyrokinetic PIC code GYGLES to address the effect of the bulk plasma temperature profile on Alfvénic stability. We find that the effect is not large at low bulk plasma beta. In this case, the coupling between the SAW and acoustic branches (with the consequent change in the SAW continuum structure and hence in the TAE frequency) is largely determined by the pressure of the fast particles. At larger bulk-plasma beta, the change in the bulk-plasma temperature profile can have considerable effect: the ballooning instability becomes the dominant one instead of the TAE mode. We have found, however, that the ballooning mode growth rate can be substantially reduced by the fast particles if their temperature is sufficiently high. We attribute this stabilizing influence to the fast-particle FLR and FOW effect [28, 29]. If the bulk-plasma temperature profile is flat, the dominant mode is the TAE instability. The main effect of the bulk-plasma temperature in this case is through the radiative and ion Landau damping mechanisms: the mode is seen to be stabilized at larger bulk-ion temperatures.

The structure of the paper is as follows. In Sec. II, the basic equations and their discretization in the code are presented. In Sec. III, the simulation results are described. The conclusions are made in Sec. IV.

\section{BASIC EQUATIONS AND NUMERICAL APPROACH}

The code solves the gyrokinetic Vlasov-Maxwell system of equations [30, 31]. The distribution function is split into a background part and a perturbation $f_{s}=F_{0 s}+\delta f_{s}$ (the index $s=i, e, f$ is used for the particle species: ions, electrons and fast particles). The background distribution function is usually taken to be a Maxwellian. The perturbed distribution 
function $\delta f_{s}$ is found from the linearized gyrokinetic Vlasov equation:

$$
\frac{\partial \delta f_{s}}{\partial t}+\dot{\mathbf{R}}^{(0)} \cdot \frac{\partial \delta f_{s}}{\partial \mathbf{R}}+\dot{v}_{\|}^{(0)} \frac{\partial \delta f_{s}}{\partial v_{\|}}=-\dot{\mathbf{R}}^{(1)} \cdot \frac{\partial F_{0 s}}{\partial \mathbf{R}}-\dot{v}_{\|}^{(1)} \frac{\partial F_{0 s}}{\partial v_{\|}} .
$$

Here, $\left[\dot{\mathbf{R}}^{(0)}, \dot{v}_{\|}^{(0)}\right]$ correspond to the unperturbed gyrocenter position and parallel velocity. $\left[\dot{\mathbf{R}}^{(1)}, \dot{v}_{\|}^{(1)}\right]$ are the perturbation of the particle trajectories proportional to the electromagnetic field fluctuations. The equations of motion are:

$$
\begin{aligned}
\dot{\mathbf{R}} & =\left(v_{\|}-\frac{q}{m}\left\langle A_{\|}\right\rangle\right) \mathbf{b}^{*}+\frac{1}{q B_{\|}^{*}} \mathbf{b} \times\left[\mu \nabla B+q\left(\nabla\langle\phi\rangle-v_{\|} \nabla\left\langle A_{\|}\right\rangle\right)\right] \\
\dot{v}_{\|} & =-\frac{1}{m}\left[\mu \nabla B+q\left(\nabla\langle\phi\rangle-v_{\|} \nabla\left\langle A_{\|}\right\rangle\right)\right] \cdot \mathbf{b}^{*}
\end{aligned}
$$

with $\phi$ and $A_{\|}$being the perturbed electrostatic and magnetic potentials, $\mu$ the magnetic moment, $m$ the mass of the particle, $B_{\|}^{*}=\mathbf{b} \cdot \nabla \times \mathbf{A}^{*}, \mathbf{b}^{*}=\nabla \times \mathbf{A}^{*} / B_{\|}^{*}, \mathbf{A}^{*}=\mathbf{A}_{0}+\left(m v_{\|} / q\right) \mathbf{b}$ the so-called modified vector potential, $\mathbf{A}_{0}$ the magnetic potential corresponding to the equilibrium magnetic field $\mathbf{B}=\nabla \times \mathbf{A}_{0}$ and $\mathbf{b}=\mathbf{B} / B$ the unit vector in the direction of the equilibrium magnetic field. The gyro-averaged potentials are defined as usual:

$$
\langle\phi\rangle=\oint \frac{\mathrm{d} \theta}{2 \pi} \phi(\mathbf{R}+\boldsymbol{\rho}),\left\langle A_{\|}\right\rangle=\oint \frac{\mathrm{d} \theta}{2 \pi} A_{\|}(\mathbf{R}+\boldsymbol{\rho}),
$$

where $\boldsymbol{\rho}$ is the gyroradius of the particle and $\theta$ is the gyro-phase. The perturbed electrostatic and magnetic potentials are found self-consistently from the gyrokinetic quasineutrality equation and parallel Ampére's law:

$$
-\nabla \cdot\left[\left(\sum_{s=i, f} \frac{q_{s}^{2} n_{s}}{T_{s}} \rho_{s}^{2}\right) \nabla_{\perp} \phi\right]=\sum_{s=i, e, f} q_{s} \delta n_{s},\left(\sum_{s=i, e, f} \frac{\hat{\beta}_{s}}{\rho_{s}^{2}}-\nabla_{\perp}^{2}\right) A_{\|}=\mu_{0} \sum_{s=i, e, f} \delta j_{\|_{s}},
$$

where $\delta n_{s}=\int \mathrm{d}^{6} Z \delta f_{s} \delta(\mathbf{R}+\boldsymbol{\rho}-\mathbf{x})$ is the perturbed gyrocenter density, $\delta j_{\|_{s}}=$ $q_{s} \int \mathrm{d}^{6} Z \delta f_{s} v_{\|} \delta(\mathbf{R}+\boldsymbol{\rho}-\mathbf{x})$ is the perturbed gyrocenter current, $q_{s}$ is the charge of the particle, $\mathrm{d}^{6} Z=B_{\|}^{*} \mathrm{~d} \mathbf{R} \mathrm{d} v_{\|} \mathrm{d} \mu \mathrm{d} \theta$ is the phase-space volume, $\rho_{s}=\sqrt{m_{s} T_{s}} /(e B)$ is the thermal gyroradius and $\hat{\beta}_{s}=\mu_{0} n_{s} T_{s} / B_{0}^{2}$ is the "partial" plasma beta corresponding to a particular species. The polarization density is treated in the long-wavelength approximation (except in Sec. III D where the generalized solver [32] is used). Finite Larmor radius (FLR) effects are neglected for electrons. The zeroth-order densities satisfy the quasineutrality equation $\sum_{s} q_{s} n_{s}=0$ with $s=i, e, f$.

The perturbed part of the distribution function is discretized with markers:

$$
\delta f_{s}\left(\mathbf{R}, v_{\|}, \mu, t\right)=\sum_{\nu=1}^{N_{p}} w_{s \nu}(t) \delta\left(\mathbf{R}-\mathbf{R}_{\nu}\right) \delta\left(v_{\|}-v_{\nu \|}\right) \delta\left(\mu-\mu_{\nu}\right),
$$


where $N_{p}$ is the number of markers, $\left(\mathbf{R}_{\nu}, v_{\nu \|}, \mu_{\nu}\right)$ are the marker phase space coordinates and $w_{s \nu}$ is the weight of a marker. The electrostatic and magnetic potentials are discretized with the finite-element method (Ritz-Galerkin scheme):

$$
\phi(\mathbf{x})=\sum_{l=1}^{N_{s}} \phi_{l} \Lambda_{l}(\mathbf{x}), \quad A_{\|}(\mathbf{x})=\sum_{l=1}^{N_{s}} a_{l} \Lambda_{l}(\mathbf{x}),
$$

where $\Lambda_{l}(\mathbf{x})$ are the finite elements (tensor product of $\mathrm{B}$ splines $[33,34]$ ), $N_{s}$ is the total number of the finite elements, $\phi_{l}$ and $a_{l}$ are the spline coefficients. A detailed description of the discretization procedure can be found in Refs. [26, 27, 32, 35, 36]. We apply the so-called phase factor transform [35] to all perturbed quantities in the code. The integrals over the gyro-angle Eq. (4) are approximated with an N-point discrete sum [32, 37, 38]. The cancellation problem $[25,26]$ is solved using the iterative scheme No. 2 described in Ref. [27].

\section{SIMULATIONS}

\section{A. Simulation parameters}

We consider a plasma with circular cross-section, but realistic magnetic field, aspect ratio and size similar to JET (Joint European Torus, see Ref. [39]): $B_{0}=3.45 \mathrm{~T}, R_{0}=3.0 \mathrm{~m}$, $\epsilon=r_{a} / R_{0}=0.333$. The magnetic equilibrium is produced solving the Grad-Shafranov equation with the CHEASE code [40] assuming the zero-pressure limit. The resulting safety factor is plotted in Fig. 1. The background magnetic field is $\mathbf{B}=\nabla \psi \times \nabla \varphi+I(\psi) \nabla \varphi$ with the toroidal angle $\varphi$, the poloidal flux $\psi$ and the poloidal current $I(\psi)$. The plasma consists of Hydrogen bulk ions, electrons and Deuterium fast particles. The unperturbed distribution functions of all particle species are Maxwellian. The bulk plasma density profile is flat. The bulk ion and electron temperature profiles are either flat or given by the expression:

$$
T_{i}(s)=T_{e}(s)=T_{0} \exp \left[-\frac{\Delta_{\mathrm{T}}}{L_{\mathrm{T}}} \tanh \left(\frac{s-s_{\mathrm{T}}}{\Delta_{\mathrm{T}}}\right)\right]
$$

where $s=\sqrt{\psi / \psi_{\mathrm{a}}}, \psi$ is the poloidal flux and $\psi_{\mathrm{a}}$ is the poloidal flux on the plasma edge. Other parameters are $s_{\mathrm{T}}=0.5$ the position of the maximal $\kappa_{\mathrm{T}}=\left|\nabla T_{i}\right| / T_{i}, T_{0}$ the bulk plasma temperature at $s=s_{\mathrm{T}}, \Delta_{\mathrm{T}}=0.2$ the "width" of the bulk plasma temperature profile, and $L_{\mathrm{T}}=0.3$ the "length" of the bulk plasma temperature profile (this parameter determines how large the temperature gradient is). The fast particle temperature $T_{f}$ is flat 
and the fast particle density is given by the expression:

$$
n_{f}(s)=n_{0 f} \exp \left[-\frac{\Delta_{\mathrm{n} f}}{L_{\mathrm{n} f}} \tanh \left(\frac{s-s_{\mathrm{n} f}}{\Delta_{\mathrm{n} f}}\right)\right]
$$

with $n_{0 f}=2 \times 10^{18} \mathrm{~m}^{-3}, s_{\mathrm{n} f}=0.5, \Delta_{\mathrm{n} f}=0.2$ and $L_{\mathrm{n} f}=0.3$.

We fix the toroidal mode number $n=-6$. For this $n$ and the safety factor profile given, a toroidicity-induced gap appears at the position of the maximal fast-particle density gradient $s=0.5$. As usual in PIC simulations, we will observe the mode which is the most unstable one for the parameters given (i.e. for the fixed toroidal mode number).

The typical numerical parameters used in the simulations are as follows: the number of ion markers $N_{i}=10^{7}$, the number of electron markers $N_{e}=4 \times 10^{7}$, the number of fast particles $N_{f}=2 \times 10^{7}$, the number of radial grid points $n_{r}=128$, the number of poloidal grid points $n_{\theta}=32$, the time step $\Delta t=2 \times 10^{-9} \mathrm{~s}$, the number of iterations in order to solve the cancellation problem $N_{\text {iter }}=4$. Convergence studies have shown that these parameters correspond to well converged simulations.

\section{B. Low-beta bulk plasma}

First, we consider the bulk plasma with a flat density $n_{0}=2 \times 10^{19} \mathrm{~m}^{-3}$ and a temperature which is either flat or given by Eq. (8) with $T_{0}=1 \mathrm{keV}$. These parameters correspond to $\beta_{\text {bulk }}=2 \mu_{0} n_{0}\left(T_{i}+T_{e}\right) / B^{2}=0.00142$. The motivation to consider that small bulk plasma beta is to minimize the effect of the coupling [16] between the shear Alfvén wave (SAW) and the acoustic branch caused by the bulk plasma pressure. However, we will see in the following that it is the fast particle pressure which determines this coupling for the parameters considered. To achieve such a low $\beta_{\text {bulk }}$, one has to take fairly small bulk plasma density (see above) so that the ratio $n_{f}(s) / n_{0} \sim 0.1$ is not very small. As a consequence, the fast-particle contribution to the plasma inertia (and hence to the Alfvén velocity) becomes non-negligible (although still minor). We have seen that both the effect of the fast particle pressure and the effect of the fast particle inertia (less important) must be included in the calculation of the ideal MHD continuum in order to understand the PIC simulation results.

We perform the PIC simulations for the parameters chosen increasing the fast particle temperature. The resulting frequency and the growth rate are shown as a function of the fastparticle temperature in Fig. 2. The case with the flat bulk plasma temperature is compared 
with the case where the bulk plasma temperature is given by Eq. (8). One can see that the effect of the bulk plasma profile is small (although there is some marginal destabilization caused by the bulk plasma temperature gradient). In Fig. 3, the time evolution is shown for $T_{f}=0.3 \mathrm{MeV}$ for both the flat and non-flat bulk plasma temperatures. Correspondingly, in Fig. 4 the eigenmode structures are presented. One sees that the resulting dependencies are very similar in both cases. This can be explained by the small value of the bulk plasma beta so that the energy content in the bulk plasma is not sufficient to change considerably the SAW spectrum. On the other hand, there is a substantial scale separation between the Alfvén frequency and the diamagnetic frequency so that the diamagnetic effect associated with the bulk plasma profile is unimportant, too. In contrast, the fast particle pressure (ranging from $\beta_{\text {fast }}=0.0135$ for $T_{f}=0.2 \mathrm{MeV}$ to $\beta_{\text {fast }}=0.0474$ for $T_{f}=0.7 \mathrm{MeV}$ ) has a strong influence on the simulation results. This can be seen from the frequency dependence on the fast particle temperature (Fig. 2). Here, the TAE gap in the MHD Alfvénic continuum is shown as it appears if only the SAW part is considered (i.e. neglecting the coupling of the SAW to the acoustic branch). One can see that the PIC frequency is within the gap for smaller $T_{f}$ indicating that the mode observed in the simulations is indeed the TAE mode. At larger fast particle temperatures, the mode frequency leaves the gap so that one can be tempted to argue that the TAE mode is modified into an Energetic Particle Mode (EPM). This, however, appears to be wrong by closer examination (although some non-perturbative effect must be present in all cases considered here). In Figs. 5 and 6, the ideal MHD continuum is shown for the cases $T_{f}=0.2 \mathrm{MeV}$ and $T_{f}=0.7 \mathrm{MeV}$ respectively (flat bulk plasma temperature). These continua are computed numerically with the eigenvalue code CONTI [41] including the coupling between the SAW and the sound mode. Both the bulk plasma and the fast particle pressures are included in the calculation (taking into account the fast particle density profile). The additional inertia resulting from the fast particle species is included, too, which has a small but non-negligible effect on the Alfvén velocity. The frequencies resulting from the PIC simulations are plotted on the continua. One sees that they still agree well with the location of the TAE gap so that the mode from the simulation is the TAE mode both for the small and the large fast particles temperatures (although, as mentioned above, this mode is perhaps modified by the fast ions; in addition, the frequency of the mode can be affected by non-ideal effects). Note that the bulk plasma properties are exactly the same both in Fig. 5 and 6 . The only difference is the fast particle temperature. 
Thus, the considerable change on the continuum structure (reshaping, frequency upshift) is caused solely by the increased fast particle pressure which determines the coupling to the acoustic branch for the parameters chosen. The fast particles affect the Alfvénic modes not only through the resonant wave-particle interaction but also by changing the MHD properties of the system (coupling to the acoustic branch) with a resulting MHD upshift in the frequency which, consequently, may affect the wave-particle resonances.

The growth rate dependence on the fast particle temperature can be explained by the combined action of the resonant fast particle destabilization (the classical $v_{\text {th } f}=v_{\mathrm{A}} / 3$ resonance [6] is pointed in Fig. 2) and the stabilizing influence of the fast particle Finite Orbit Width (FOW) effects [7] (see also Ref. [8] for a related discussion).

\section{High-beta bulk plasma}

We consider Alfvénic modes in the same magnetic configuration as in the previous Section but at high bulk-plasma beta. These modes are destabilized by the energetic particles with the same parameters as described above. The bulk plasma temperature is either flat or given by Eq. (8) with $T_{0}=3 \mathrm{keV}$. This larger (comparing with Sec. III B) bulk plasma temperature leads to increased radiative damping (see the discussion in the next section). The bulk plasma density $n_{0}=2 \times 10^{20} \mathrm{~m}^{-3}$ corresponds to considerably larger $\beta_{\text {bulk }}=0.041$. Clearly, for this bulk plasma beta, the energetic particle inertia is negligible compared with that of the bulk ions. The energetic particle pressure is comparable to the bulk plasma pressure. This will have consequences for the MHD continuum: changing the parameters of the energetic particles one changes also the structure of the continuum if the energetic particle pressure is large enough (in a way similar to the previous section). In contrast with the previous section (low $\beta_{\text {bulk }}$ ), one can expect a substantial effect of the bulk-plasma temperature profiles on the Alfvénic modes. In what follows we will see that it is indeed the case.

We start with a simulation using the fast particle temperature $T_{f}=0.3 \mathrm{MeV}$. The resulting time signals are shown in Fig. 7 for the case with the flat bulk-plasma temperature (on the left) and the temperature profile given by Eq. (8). One can see that the frequency is much smaller and the mode drive is much larger in the case where Eq. (8) is used. The eigenmode structure is shown in Fig. 8. One can see that the mode on the left (correspond- 
ing to the flat bulk plasma temperature) has characteristic fine scale structures around its maximum indicating an increased role of non-ideal effects (qualitatively similar structures have been previously observed with other gyrokinetic codes, e.g. LIGKA [19] and, recently, with GEM [42]). Interestingly, the size of these structures (few centimetres) is comparable to the fast-particle gyroradius. The mode on the right has a frequency in the acoustic range and is strongly driven. In what follows we will see that this mode is predominantly driven by the bulk plasma pressure gradient. The effect of the fast particles can be both destabilizing and even stabilizing depending on the parameters (fast-particle temperature) chosen. The dependence of the mode frequency and the growth rate is shown in Fig. 9 for the case with the flat bulk plasma temperature profile. The most unstable mode in this case is the TAE mode. Similarly to the case with low $\beta_{\text {bulk }}$, the frequency increases with the fast-particle temperature (pressure). This is caused by the MHD continuum upshift and reshaping with increasing fast-particle pressure. The changes in the MHD continuum occur due to the coupling between the acoustic and SAW modes [16]. This coupling can be influenced considerably by the fast-particle population provided the fast-particle beta is large enough (i.e. comparable to $\beta_{\text {bulk }}$ ). In Fig. 10, the MHD continuum corresponding to the case with $T_{f}=0.7 \mathrm{MeV}$ and flat bulk plasma temperature profile is shown. One sees that the frequency resulting from the PIC simulations is in a quite reasonable agreement with the location of the TAE gap although the gap structure and the entire continuum have been strongly reshaped and shifted by the combined effect of the bulk-plasma and the fast-particle pressure (both act on equal footing and determine the coupling to the acoustic branch).

The growth rate dependence on $T_{f}$ is determined, as usual, by the combined effect of the resonant interaction of the fast particles with the wave and the stabilizing influence of the fast-particle finite orbit width [7] (becoming more important at larger $T_{f}$ ). It is interesting that in contrast to the low beta case (i.e. large Alfvén velocity), the resonance $v_{\operatorname{thf}}=v_{\mathrm{A}}$ [6] appears to be more important at the large $\beta_{\text {bulk }}$ because this resonance corresponds to practically relevant fast-particle temperatures whereas the resonance $v_{\text {th } f}=v_{\mathrm{A}} / 3$ (most important at low $\beta_{\text {bulk }}$ ) takes place at much smaller $T_{f}$ (the mode is stable at such small $T_{f}$ because the fast particle drive is proportional to $k_{\perp} \rho_{f}$ with $\rho_{f}$ the fast-particle gyroradius). Interestingly, the growth rate peaks at $v_{\operatorname{th} f}$ somewhat less than $v_{\mathrm{A}}$ (but much larger than $\left.v_{\mathrm{A}} / 3\right)$ indicating perhaps the optimal relation between the resonant drive and the fast-ion 


\section{FOW stabilization.}

Finally, consider Fig. 11. Here, the dependence of the mode growth rate on the fast particle temperature is presented for the case with the bulk plasma profile given by Eq. (8). In this case, one sees that the mode is already unstable in the absence of the fast particles (see the dashed straight line). Applying the local ideal ballooning mode stability criterion for large-aspect-ratio circular cross section plasma $-r \beta^{\prime}<(2 / 3 \hat{s}) r /\left(R q^{2}\right)$ [43], we find that the parameters chosen are in the unstable domain (here, $\hat{s}$ is the magnetic shear, $\beta$ is the bulk-plasma beta, $r$ is the small radius, $R$ is the large radius and $q$ is the safety factor). We conclude that the mode observed in our simulations should be a ballooning instability destabilized by the bulk plasma pressure gradient. As expected, the frequency of this mode is very small (although finite). The role of the fast particles in the mode stability depends, as one can see, on a particular choice of the fast particle temperature. At lower $T_{f}$ a further resonant destabilization of the already unstable ballooning mode is possible. At larger $T_{f}$, the mode is stabilized by the fast particles. The stabilization becomes even larger if the fast particle density is increased (see the line with circles which corresponds to the fast particle density $n_{0 f}=4 \times 10^{18} \mathrm{~m}^{-3}$ ). This stabilization can probably be associated with the fast-ion finite orbit width effect $[28,29]$ which is more pronounced at larger temperatures. Another possible explanation is large magnetic drift velocity of the fast particles which decouples the hot species from ballooning modes $[29,44]$.

The simulations presented in Fig. 11 have two drawbacks. First, the vacuum equilibrium magnetic field has been used. Clearly, the equilibrium corrections associated with the finite pressure are important for the ballooning mode physics. Second, the fast-particle beta is unrealistic (too large) at the large fast-particle temperatures $\left(T_{f} \geq 1.5 \mathrm{MeV}\right)$. Hence, we consider the result presented in Fig. 11 as a preliminary one which nevertheless indicates an important physics which clearly deserves a further study. The stabilizing influence of the fast particles on MHD modes is in particular interesting (in terms of practical relevance). As a possible application, one has imagined heating of some groups of ions in certain MHD unstable regions of the plasma with the consequent energetic-particle stabilization of these MHD modes (see Ref. [44]). Further study of the kinetic effects on the MHD ballooning modes is needed to access various important effects such as the FLR and FOW effects associated with both the fast-particle species and the bulk plasma. We plan to perform such a study in a systematic way using the gyrokinetic PIC simulations in the near future. 


\section{Radiative damping}

Above, we have considered the role of the bulk plasma temperature profile comparing the flat temperature with the profile given by Eq. (8). In what follows, we keep the bulk plasma profiles flat while changing the value of the temperature (ion and electron temperatures are always equal to each other). We consider a high-beta plasma with the parameters described in the previous section. The fast-particle density $n_{0 f}=2 \times 10^{18} \mathrm{~m}^{-3}$ and the fast-particle temperature $T_{f}=0.4 \mathrm{MeV}$ (this corresponds to $\beta_{\text {fast }}=0.027$ ). In Fig. 12, the frequency and the growth rate resulting from the PIC simulations are plotted. One can see that the mode frequency increases with the bulk plasma temperature. This tendency has already been discussed; it is related to the continuum upshift and reshaping which is however now caused by the bulk plasma pressure (because the fast-particle parameters are fixed). For example, Fig. 13 shows the continuum for the case $T_{i}=5 \mathrm{keV}$. One can see that the frequency found in the PIC simulations indeed remains within the TAE gap although its absolute value has clearly increased from $T_{i}=1 \mathrm{keV}$.

The mode growth rate decreases substantially with the bulk plasma temperature. We attribute this to the enhanced radiative damping at larger ion temperatures (i.e. larger thermal gyroradius). Similarly, there is a strong stabilization effect (not shown here) when the magnetic field is decreased.

Historically, radiative damping has been introduced as an FLR correction associated with higher order contributions $\left(\sim k_{\perp}^{4} \rho_{i}^{4}\right)$ to the polarization density (see Ref. [15] for example). In Fig. 12, we compare simulations using the so-called generalized solver [32] which employs the polarization density exact to all orders in $k_{\perp} \rho_{i}$ (detailed description in Ref. [32]) and simulations using the so-called long-wavelength approximation of the polarization density (accurate only to $k_{\perp}^{2} \rho_{i}^{2}$ ). We see that the effect of the higher-order contributions to the polarization density is visible but not large. Apparently, these are the terms associated with the gyro-average in the equations of the motion and in the Vlasov equation which produce the radiative damping in the case considered. Note that the bulk-ion gyroradius changes from $k_{\theta} \rho_{i} \approx 2 m \rho_{i} / r_{a}=0.018$ for $T_{i}=1 \mathrm{keV}$ to $k_{\theta} \rho_{i} \approx 0.04$ for $T_{i}=5 \mathrm{keV}$ (here, $m=10$, $r_{a}=1 \mathrm{~m}$ is the minor radius and the factor 2 accounts for the mode location approximately on the half minor radius). It is interesting that even for these rather small values of $k_{\theta} \rho_{i}$, the radiative damping is an important mechanism (which can be described rather accurately 
within the long-wavelength approximation of the polarization density). Of course, the radial small-scale structures (similar to shown in Fig. 8) will increase the total perpendicular wave number and, hence, the role of the bulk-ion FLR effects.

On the other hand, one could argue that the ion Landau damping increases as well with the bulk-ion temperature and that the stabilising effect observed in Fig. 12 can be explained by this mechanism rather than the larger radiative damping at larger $T_{i}$. Then, the discussion about higher-order terms in the polarization density would become irrelevant because they do not influence the Landau damping. To check this hypothesis, we perform the simulations shown in Fig. 14. Here, we fix the bulk-ion temperature $T_{i}=1 \mathrm{keV}$ and decrease the bulk-ion charge number $Z_{i}$ so that the resulting bulk-ion gyroradius $\rho_{i} \propto \sqrt{T_{i}} / Z_{i}$ increases in the same way as in Fig. 12 where the bulk-ion temperature increased while $Z_{i}$ was constant (note that the electron charge must be changed simultaneously $Z_{e}=Z_{i}$ in order to provide the ambient quasineutrality at the same bulk-plasma beta). One sees that the frequency remains constant if $T_{i}$ is constant. Clearly, the pressure does not change. Hence, the acoustic coupling and the associated frequency upshift remain unchanged. In contrast, the growth rate decreases almost identically to the case where $T_{i}$ changes but the bulk-ion charge number is constant. This indicates that the damping associated with the bulk-ion FLR effect (i.e. the radiative damping) is indeed the dominant mechanism for the case considered. Another simulation which supports this conclusion is shown in Fig. 15. Here, we change the bulk-ion temperature and the charge number simultaneously so that the ratio $T_{i} / Z_{i}^{2}=5 \mathrm{keV}$ is kept constant (and hence the gyroradius). The result is again straightforward to interpret. The frequency changes as it does in the case with the fixed $Z_{i}=1$ (because the plasma pressure increases). The growth rate, in contrast, remains essentially constant (because the bulk-ion FLR effect is fixed). This demonstrates that the radiative damping (bulk-ion FLR) plays a dominant role in the mode stabilisation.

Summarising, we have shown that our initial assumption about the dominant role of the radiative damping in the mode stabilisation observed in Fig. 12 was correct. We have also seen that the long-wavelength approximation is good enough for the case considered. The ion FLR effect needed for the radiative damping must be related to the gyro-average procedure (carried to higher orders in $k_{\perp} \rho_{i}$ ) in the equations of motion and the Vlasov equation. 


\section{CONCLUSIONS}

The global gyrokinetic particle-in-cell code GYGLES has been used to address the effect of the plasma pressure on Alfvénic modes. It has been found that both the bulk-plasma pressure and the fast-particle pressure are important for the structure of the SAW continuum. For example, increasing the fast-particle pressure can substantially shift and reshape the SAW continuum (if the fast-particle beta is comparable to the bulk-plasma one). As consequence, the TAE gap is shifted to higher frequencies and hence, the TAE frequency increases (this should be possible to see experimentally). Another quantity which determines the frequency of the unstable TAE mode is the Alfvén velocity itself which depends on the entire inertia of the plasma. Usually, the bulk ions make the dominant contributions to the plasma inertia. One can, however, imagine situations where other species make non-negligible contributions to the plasma inertia (e.g. some heavy impurity). This might lead to a modification in the SAW continuum which perhaps can be assessed measuring the associated change in the frequency of the unstable TAE mode. The subject deserves further studies.

Another interesting effect observed in this paper is the fast-particle stabilization of the ballooning modes. The mode is stabilized if the fast-particle temperature is large enough. We attribute this to the energetic-ion FLR and FOW effects [28]. In practice, this effect could be used (as suggested in Ref. [44]) to stabilize the MHD unstable modes heating certain groups of particles in the regions of the plasma where the ballooning mode activity is enhanced. This might help to increase the beta limit in tokamaks.

The bulk-ion FLR and FOW effects must be considered, too. These effects cause, for example, the radiative damping which was relatively high in the simulations presented in this paper. The radiative damping is increased with the bulk-plasma temperature and becomes smaller with the ambient magnetic field. Thus, one might expect a reduced radiative damping of the TAE mode (i.e. enhanced instability) for the International Tokamak Experimental Reactor (ITER) [45] parameters compared with present-day experiments (such as JET). This question will be addressed in future.

There are several other issues which remain to be studied. First, isotropic Maxellian distribution functions were used for fast particles in this paper. This appears to be sufficient in order to address the basic behaviour of Alfvénic modes interacting with fast particles (modelled using a particle-in-cell code). However, in reality the fast-ion population is usually 
better described as a slowing-down distribution (in some cases anisotropic). This has to be taken into account when real experimental configurations are modelled. Another issue is related to the Shafranov shift which has not been included in this paper. It is well known that the Shafranov shift has an important stabilizing effect on the ballooning modes. This should also be the case for the TAE stability. For the ballooning modes, the bulk-plasma pressure gradient destabilizes a mode and, at the same time, causes the Shafranov shift which is usually stabilizing. Similarly, for the TAE modes, the fast-particle pressure gradient would destabilize the TAE mode and cause an additional stabilizing Shafranov shift (providing that the fast-particle pressure is not too small). In our paper, we have included only the "destabilising part" of the pressure gradient effect, both for the bulk plasma and for the fast particles. In future, we plan to use the VMEC code [46] in order to construct the magnetic equilibria which would give us much more flexibility in this respect (there is also a version of the code which allows to treat effects of the pressure anisotropy caused e. g. by fast particles on the equilibrium [47]).

\section{ACKNOWLEDGEMENTS}

We acknowledge the support of P. Helander and S. Günter for this work. CRPP Lausanne and in particular O. Sauter are acknowledged for providing the CHEASE code. Most of the simulations are performed on the HPC-FF (Forschungszentrum Jülich) and Blue Gene/P (Rechenzentrum Garching) supercomputers. Support of the HLST group (Garching) on the code optimization is appreciated.

[1] M. N. Rosenbluth and P. H. Rutherford, Phys. Rev. Lett. 34, 1428 (1975).

[2] F. Zonca, S. Briguglio, L. Chen, C. Fogaccia, T. S. Hahm, A. V. Milovanov, and G. Vlad, Plasma Phys. Controlled Fusion 48, B15 (2006).

[3] W. W. Heidbrink, Phys. Plasmas 15, 055501 (2008).

[4] G. Y. Fu and J. W. V. Dam, Phys. Fluids B 1, 1949 (1989).

[5] J. Candy and M. N. Rosenbluth, Plasma Phys. Controlled Fusion 35, 957 (1993). 
[6] L. Chen, Phys. Plasmas 1, 1519 (1994).

[7] N. N. Gorelenkov, C. Z. Cheng, and G. Y. Fu, Phys. Plasmas 6, 2802 (1999).

[8] A. Mishchenko, A. Könies, and R. Hatzky, Phys. Plasmas 16, 082105 (2009).

[9] G. Y. Fu and C. Z. Cheng, Phys. Fluids B 4, 3722 (1992).

[10] F. Zonca and L. Chen, Phys. Rev. Lett. 68, 592 (1992).

[11] H. L. Berk, J. W. V. Dam, Z. Guo, and D. M. Lindberg, Phys. Fluids B 4, 1806 (1992).

[12] M. N. Rosenbluth, H. L. Berk, J. W. V. Dam, and D. M. Lindberg, Phys. Fluids B 4, 2189 (1992).

[13] R. R. Mett and S. M. Mahajan, Phys. Fluids B 4, 2885 (1992).

[14] H. L. Berk, R. R. Mett, and D. M. Lindberg, Phys. Fluids B 5, 3969 (1993).

[15] J. Candy and M. N. Rosenbluth, Phys. Plasmas 1, 356 (1994).

[16] M. S. Chu, J. M. Green, L. L. Lao, A. D. Turnbull, and M. S. Chance, Phys. Fluids B 4, 3713 (1992).

[17] A. B. Mikhailovskii and I. G. Shuchman, Zh. Eksp. Teor. Fiz. 71, 1813 (1976).

[18] F. Zonca, L. Chen, and R. Santoro, Plasma Phys. Controlled Fusion 38, 2011 (1996).

[19] P. Lauber, S. Günter, and S. D. Pinches, Phys. Plasmas 12, 122501 (2005).

[20] P. Lauber, S. Günter, A. Könies, and S. D. Pinches, J. Comp. Phys. 226, 447 (2007).

[21] Y. Nishimura, Z. Lin, and W. X. Wang, Phys. Plasmas 14, 042503 (2007).

[22] Y. Nishimura, Phys. Plasmas 16, 030702 (2009).

[23] J. Lang, Y. Chen, S. Parker, and G.-Y. Fu, Phys. Plasmas 16, 102101 (2009).

[24] A. Mishchenko, R. Hatzky, and A. Könies, Phys. Plasmas 15, 112106 (2008).

[25] Y. Chen and S. Parker, J. Comp. Phys 189, 463 (2003).

[26] A. Mishchenko, R. Hatzky, and A. Könies, Phys. Plasmas 11, 5480 (2004).

[27] R. Hatzky, A. Könies, and A. Mishchenko, J. Comp. Phys. 225, 568 (2007).

[28] D. A. Spong, D. J. Sigmar, W. A. Cooper, D. E. Hastings, and K. T. Tsang, Phys. Fluids 28, $2494(1985)$.

[29] P. J. Catto, R. J. Hastie and J. W. Connor, Plasma Phys. Controlled Fusion 27, 307 (1985).

[30] T. S. Hahm, Phys. Fluids 31, 2670 (1988).

[31] T. S. Hahm, W. W. Lee, and A. J. Brizard, Phys. Fluids 31, 1940 (1988).

[32] A. Mishchenko, A. Könies, and R. Hatzky, Phys. Plasmas 12, 062305 (2005).

[33] C. de Boor, A Practical Guide to Splines (Springer-Verlag, New York, 1978). 
[34] K. Höllig, Finite Element Methods with B-Splines (Society for Industrial and Applied Mathematics, Philadelphia, 2003).

[35] M. Fivaz, S. Brunner, G. de Ridder, O. Sauter, T. M. Tran, J. Vaclavik, L. Villard, and K. Appert, Comp. Phys. Commun. 111, 27 (1998).

[36] A. Mishchenko, A. Könies, and R. Hatzky, in Proc. of the Joint Varenna-Lausanne International Workshop (Società Italiana di Fisica, Bologna, 2004).

[37] W. W. Lee, J. Comp. Phys. 72, 243 (1987).

[38] R. Hatzky, T. M. Tran, A. Könies, R. Kleiber, and S. J. Allfrey, Phys. Plasmas 9, 898 (2002).

[39] P. H. Rebut and B. E. Keen, Fusion Technol. 11, 13 (1987).

[40] H. Lütjens, A. Bondeson, and O. Sauter, Comp. Phys. Commun. 97, 219 (1996).

[41] A. Könies, A. Mishchenko, and R. Hatzky, in Proc. of the Joint Varenna-Lausanne International Workshop (AIP Conference Proceedings, New York, 2008).

[42] Y. Chen, S. Parker, J. Lang, and G.-Y. Fu, Phys. Plasmas 17, 102504 (2010).

[43] J. Freidberg, Rev. Mod. Phys. 54, 801 (1982).

[44] M. N. Rosenbluth, S. T. Tsai, J. W. Van Dam, adn M. G. Engquist, Phys. Rev. Lett. 51, 1967 (1983).

[45] Editors of "Progress in the ITER Physics Basis", Nucl. Fusion 47, S1 (2007).

[46] S. P. Hirshman and J. C. Whitson, Phys. Fluids 26, 3553 (1983).

[47] W. A. Cooper, J. P. Graves, S. P. Hirshman, P. Merkel, J. Kisslinger, H. F. G. Wobig, K. Y. Watanabe and Y. Narushima, AIP Conf. Proc. 1069, 40 (2008). 


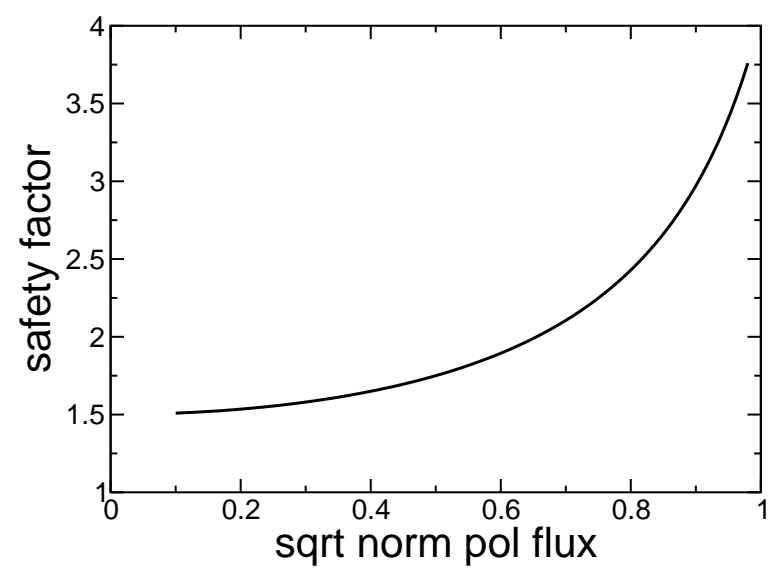

FIG. 1: Safety factor resulting from the solution of the Grad-Shafranov equation (zero-pressure limit) using the code CHEASE [40]. 

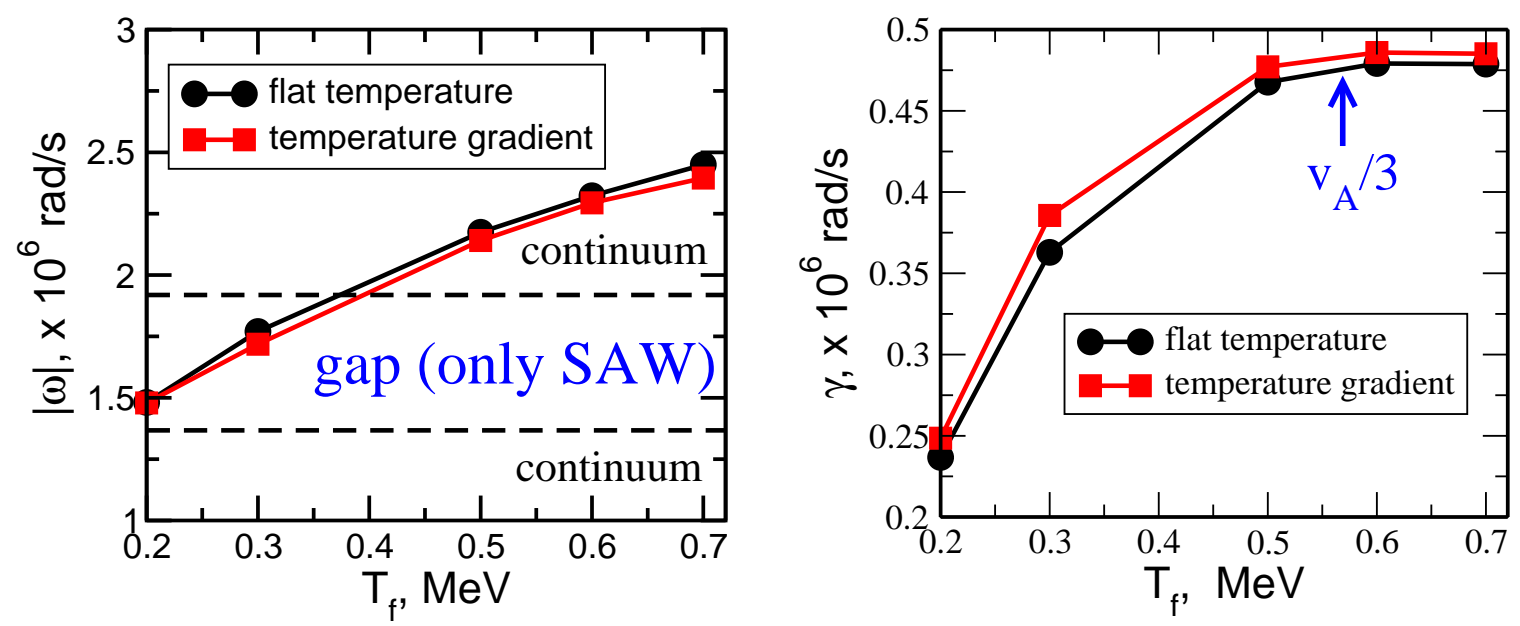

FIG. 2: (Color online): The frequency (left) and the growth rate (right) as a function of the fastparticle temperature at low bulk-plasma beta $\left(\beta_{\text {bulk }}=0.00142\right)$. The effect of the bulk-plasma temperature gradient $\left[L_{\mathrm{T}}=0.3\right.$, see Eq. (8) for details $]$ is small in this parameter range. One sees that the frequency increases with the fast-particle temperature. This frequency upshift is caused by the fast-particle pressure and is analogous to the upshift resulting from the acoustic coupling [16] when the bulk-plasma beta is taken into account. 

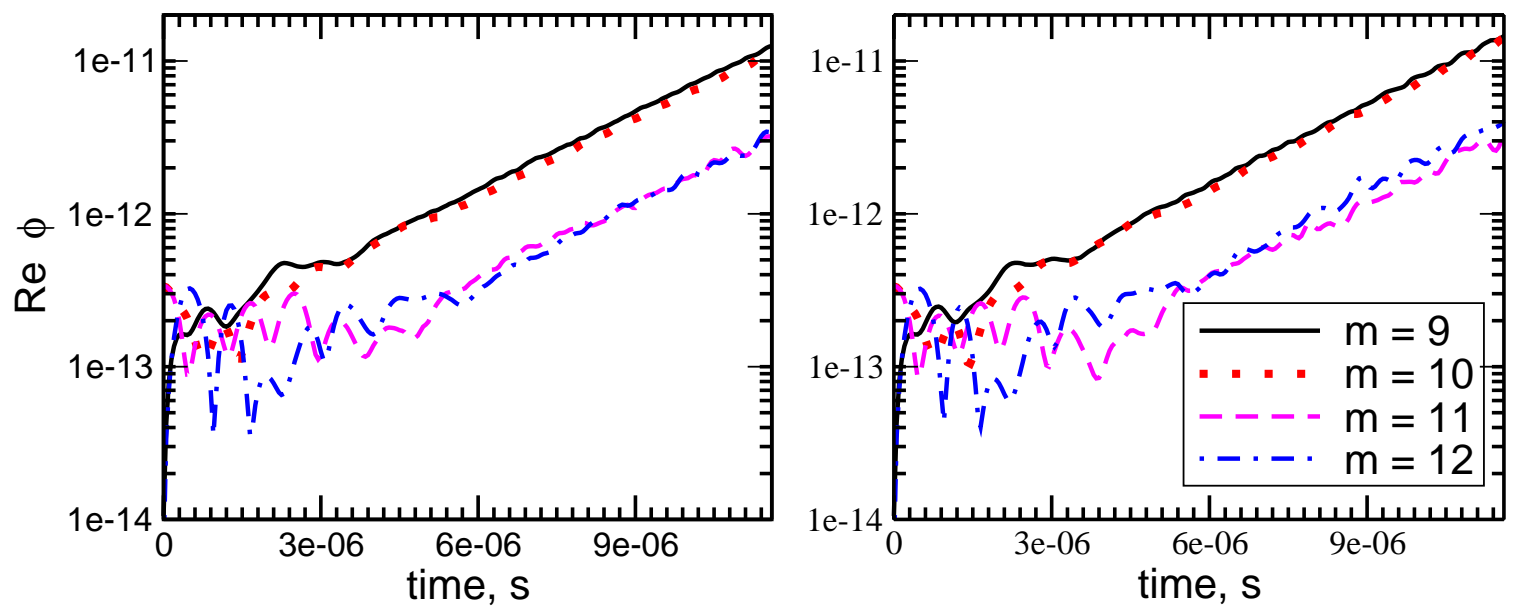

FIG. 3: (Color online): TAE mode evolution at low bulk-plasma beta; flat bulk-plasma temperature on the left and the bulk-plasma temperature profile given by Eq. (8) on the right. The fast-particle temperature $T_{f}=0.3 \mathrm{MeV}$. One sees that the difference between the signals is not large. 

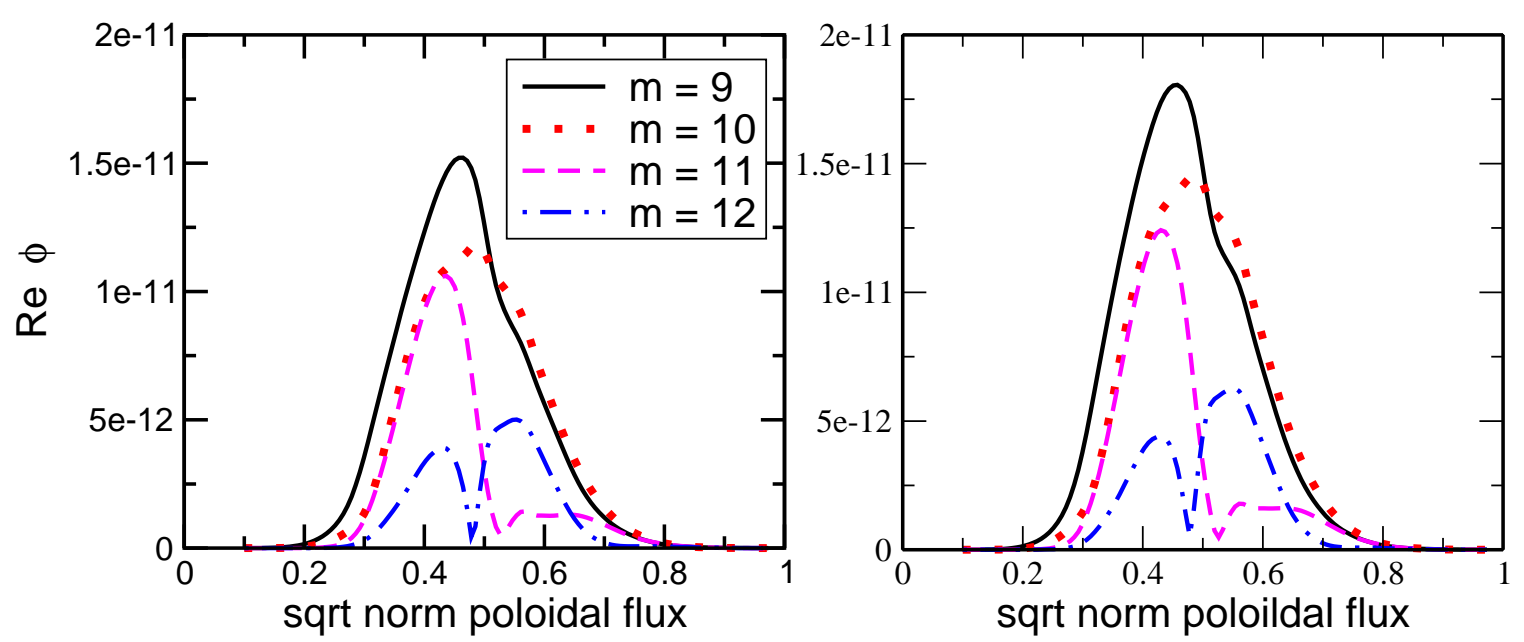

FIG. 4: (Color online): On the left: TAE mode structure at low bulk-plasma beta and flat bulkplasma temperature. On the right: TAE mode structure with finite bulk-plasma temperature profile given by Eq. (8). The fast-particle temperature $T_{f}=0.3 \mathrm{MeV}$. One sees that the difference is rather small, i.e. the effect of the bulk-plasma temperature is not large at low $\beta_{\text {bulk }}$. Both radial patterns are measured at the same simulation time. 


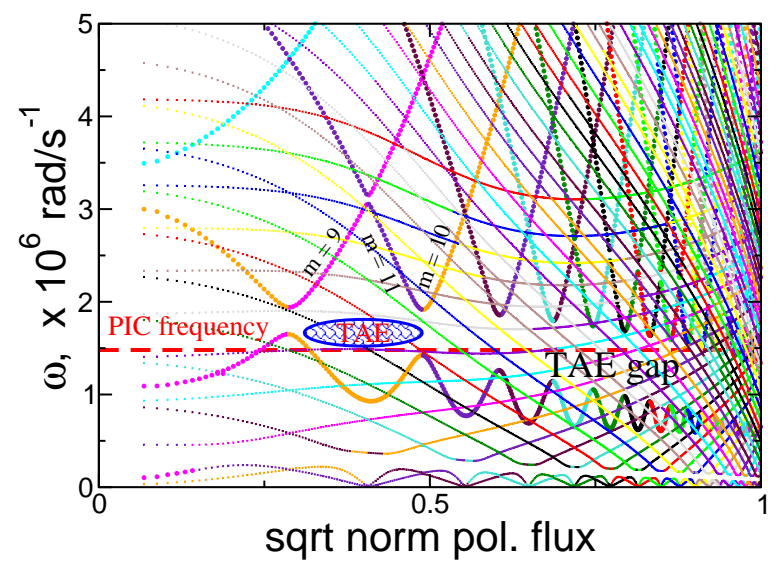

FIG. 5: (Color online): The SAW-acoustic continuum corresponding to $T_{f}=0.2 \mathrm{MeV}$ in Fig. 2 . The frequency resulted from the PIC simulations and the eigenmode location relative to the continuum are plotted, too (the red dashed line and the blue ellipse). This frequency is within the TAE gap. 


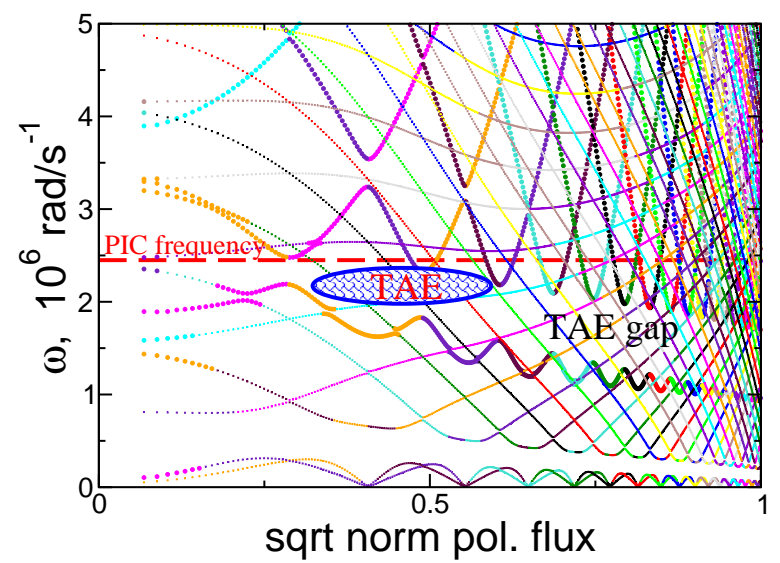

FIG. 6: (Color online): The SAW-acoustic continuum corresponding to $T_{f}=0.7 \mathrm{MeV}$ in Fig. 2 . The frequency resulted from the PIC simulations and the eigenmode location relative to the continuum are plotted, too (the red dashed line and the blue ellipse). This frequency is within the TAE gap. The SAW continuum is shifted and reshaped compared with Fig. 5. This shift is caused by the enhanced coupling between the SAW and the acoustic branch [16] caused by the enhanced fast-particle beta and, consequently, the increased total plasma pressure. 

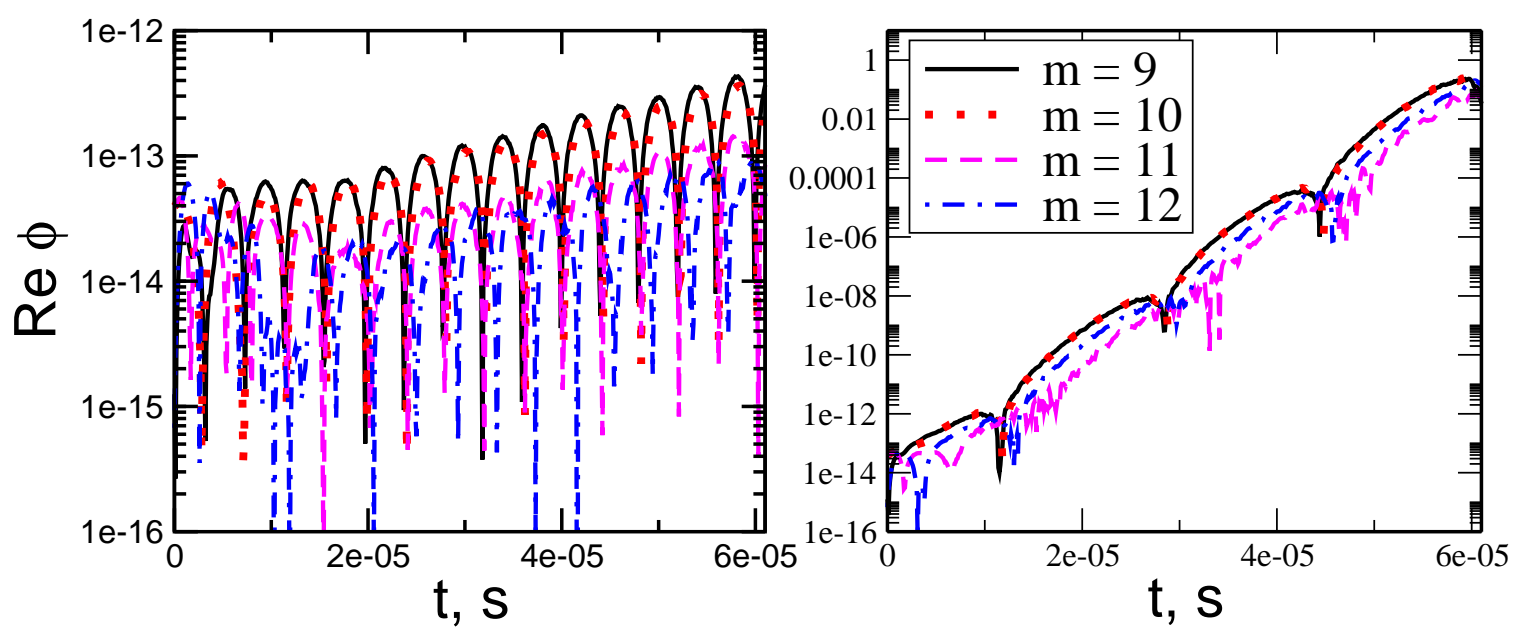

FIG. 7: (Color online): The fast-particle destabilized mode $\left(T_{f}=3 \mathrm{keV}\right)$. On the left: the bulk plasma temperature profile is flat. The mode observed is the TAE. On the right: the bulk plasma temperature profile is given by Eq. (8). The mode is stronlgy driven compared with the case with the flat bulk-plasma temperature profile. Its frequency is much smaller. This is a ballooning mode. 

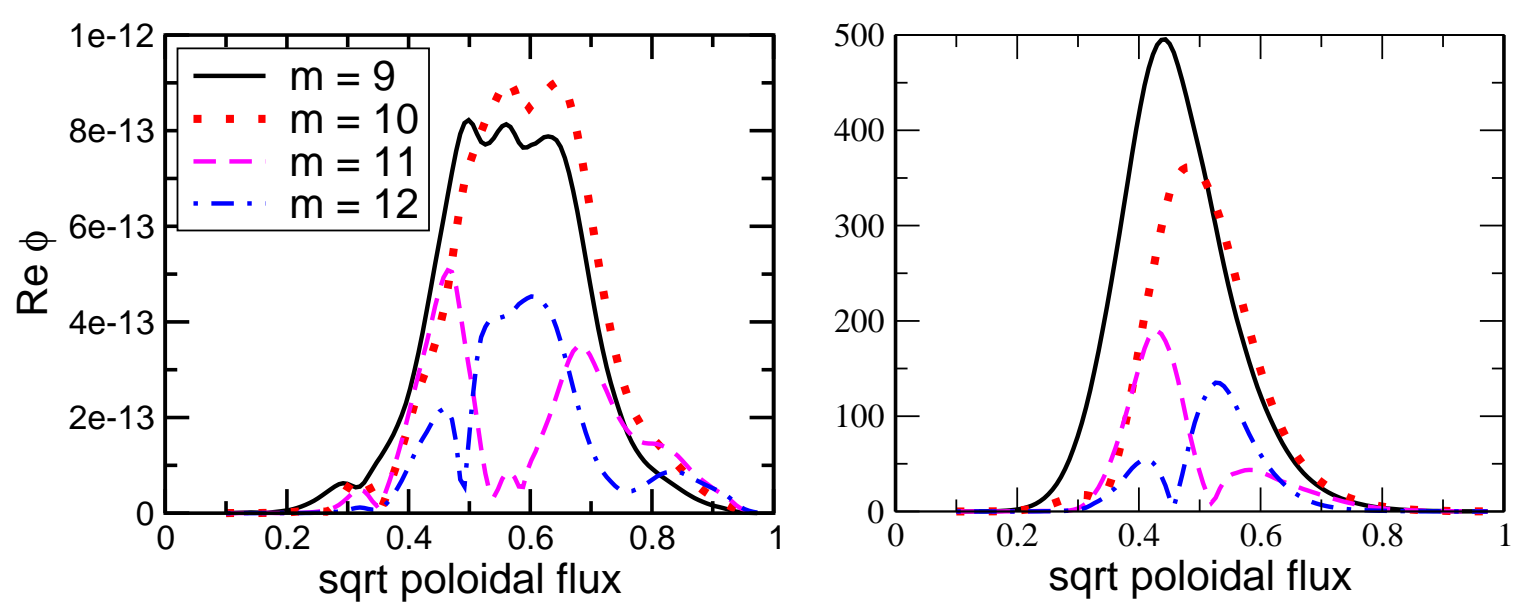

FIG. 8: (Color online): The fast-particle destabilized mode $\left(T_{f}=0.3 \mathrm{MeV}\right)$. Both radial patterns are obtained at the same simulation time $t=0.713 \times 10^{-4} \mathrm{~s}$. On the left: the bulk-temperature profile is flat (TAE mode). One can see the typical FLR structures corresponding to the mode conversion (radiative damping of the TAE mode). On the right: the bulk-plasma temperature profile is given by Eq. (8). The radiative-damping structures are absent. The mode is stronglydriven (cf. the amplitudes) and has the much smaller frequency (see Fig. 7). This is a ballooning mode. 

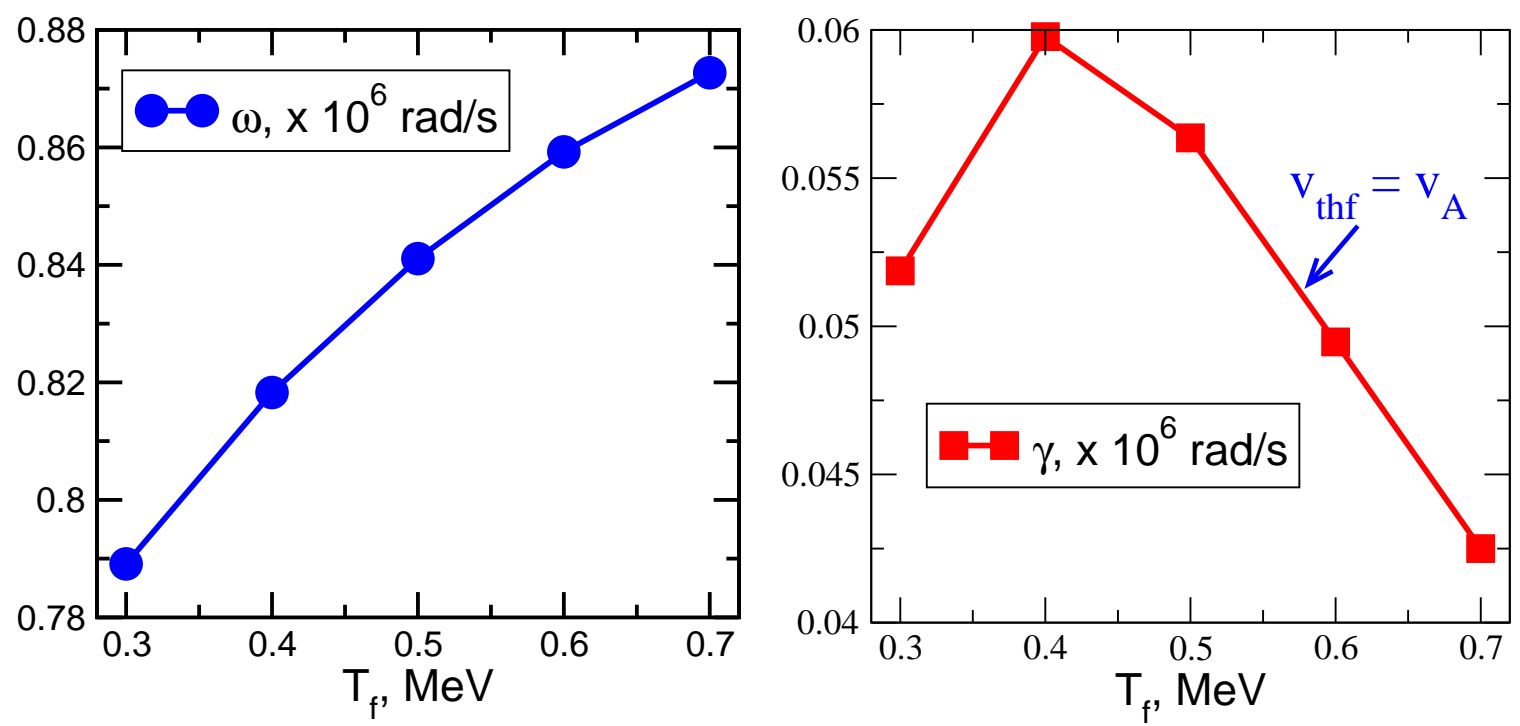

FIG. 9: (Color online): The frequency (left) and the growth rate (right) of the TAE mode in the high-beta plasma. The bulk-plasma temperature profile is flat. The $v_{\text {th } f}=v_{\mathrm{A}}$ resonance [6] is shown. 


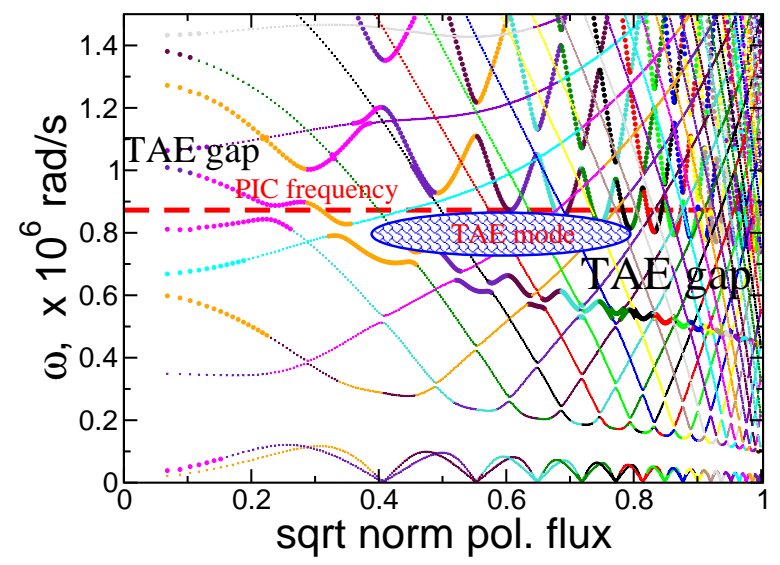

FIG. 10: The MHD continuum, the frequency resulting from the PIC simulations (the red dashed line) and the eigenmode location (the blue ellipse) corresponding to large $\beta_{\text {bulk }}, T_{f}=0.7 \mathrm{MeV}$ and the flat bulk plasma temperature profile. The mode frequency is within the TAE gap. 


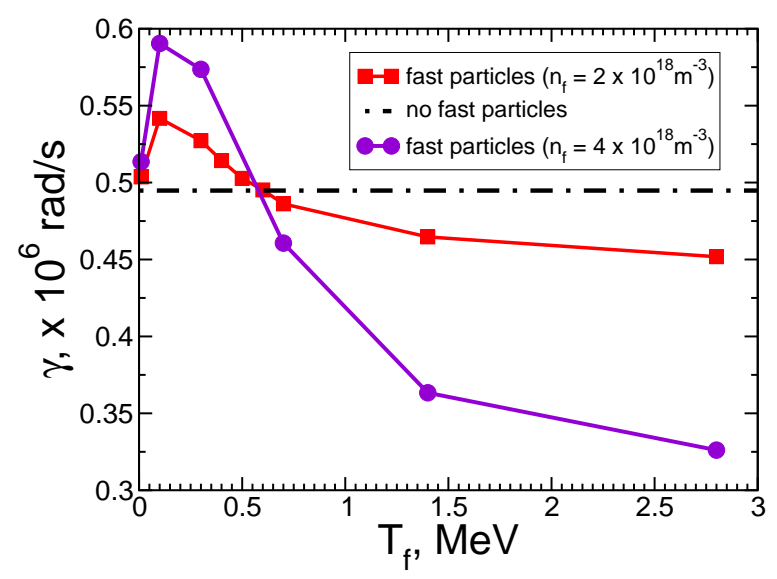

FIG. 11: (Color online): The growth rate of the ballooning mode as a function of the fast particle temperature. The black dashed line denotes the mode growth rate in the absence of the fast particles. The line with the squares corresponds to the fast-particle density $n_{0 f}=2 \times 10^{18} \mathrm{~m}^{-3}$ and the line with the circles to the fast-particle density $n_{0 f}=4 \times 10^{18} \mathrm{~m}^{-3}$. One sees that the stabilizing influence increases with the fast particle density. 

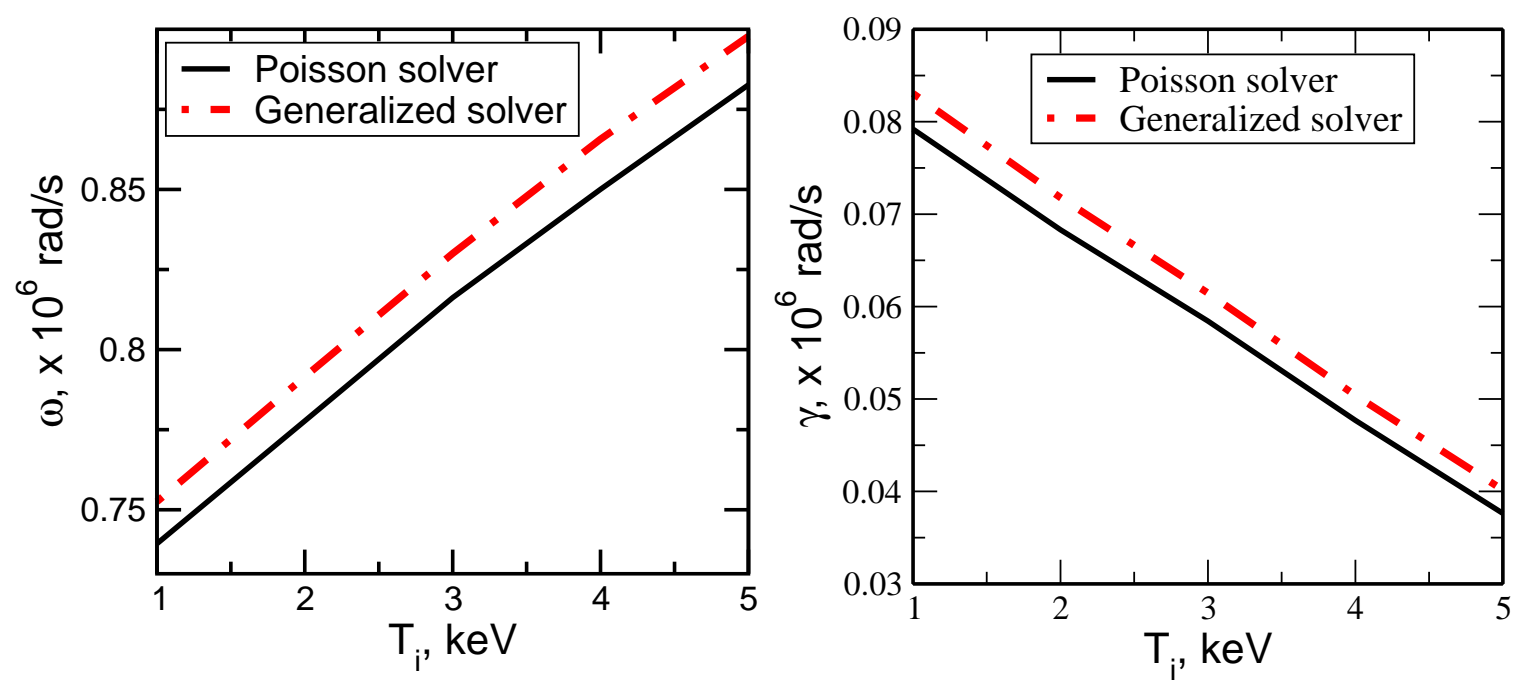

FIG. 12: (Color online): The frequency (on the left) and the growth rate (on the right) as a function of the bulk plasma temperature (flat profile). The long-wavelength approximation of the polarization density (Poisson solver) is compared with the exact expression for the polarization density (generalized solver, see Ref. [32]). 


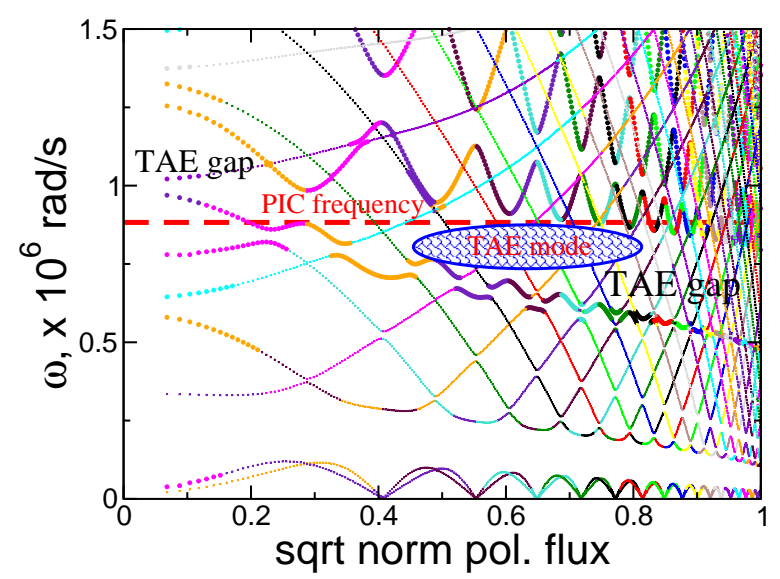

FIG. 13: (Color online): MHD continuum structure corresponding to $T_{i}=5 \mathrm{keV}$. The mode frequency resulting from the PIC simulations and the eigenmode locations are plotted, too (the red dashed line and the blue ellipse). The PIC frequency is within the TAE gap. The coupling between the acoustic and the SAW branches must be taken into account. 

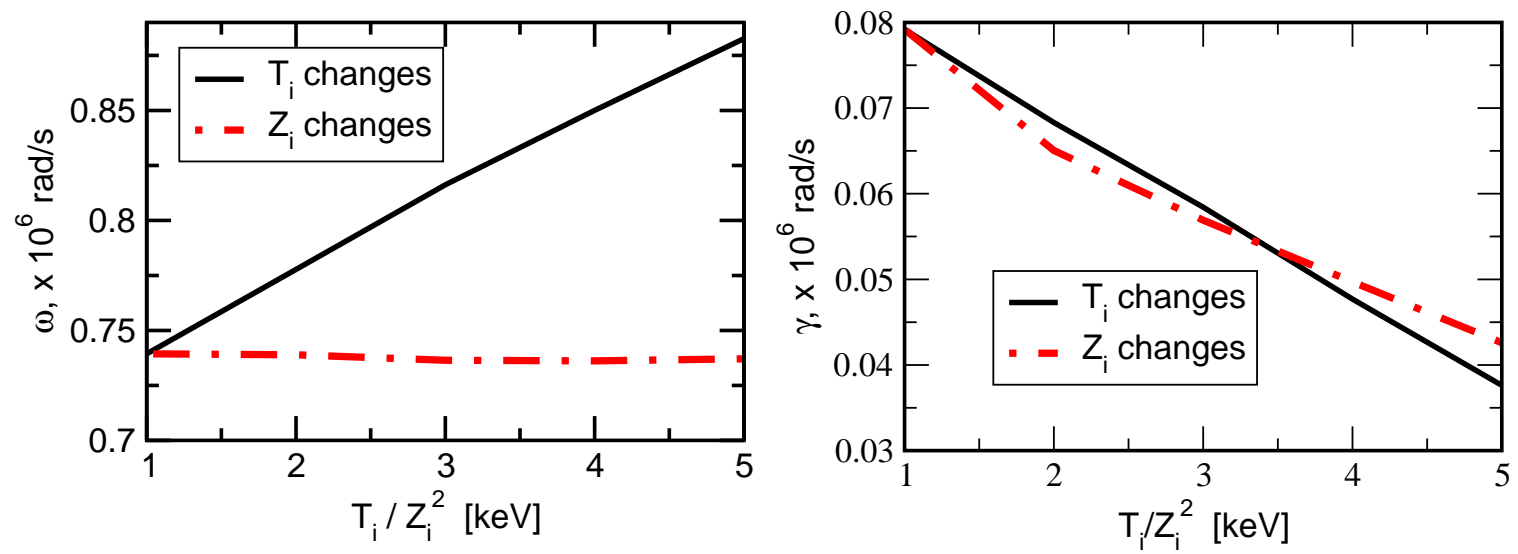

FIG. 14: (Color online): The frequency (on the left) and the growth rate (on the right) as a function of the bulk plasma temperature (flat profile) divided by the squared bulk-ion charge number. The solid line corresponds to the case when the bulk-ion temperature increases while the bulk-ion charge number is kept constant. For the dashed line, the bulk-ion temperature is kept constant $T_{i}=1 \mathrm{keV}$ (i.e. fixed ion Landau damping) while the charge number decreases (hence the gyroradius and the radiative damping increase). One sees that the damping rate is almost the same for both lines although the ion Landau damping is fixed for the dashed line and increases with the temperature for the solid line. This signalises that the radiative damping (i.e. the damping related to the bulk-plasma FLR) is the dominant mechanism for the case considered. 

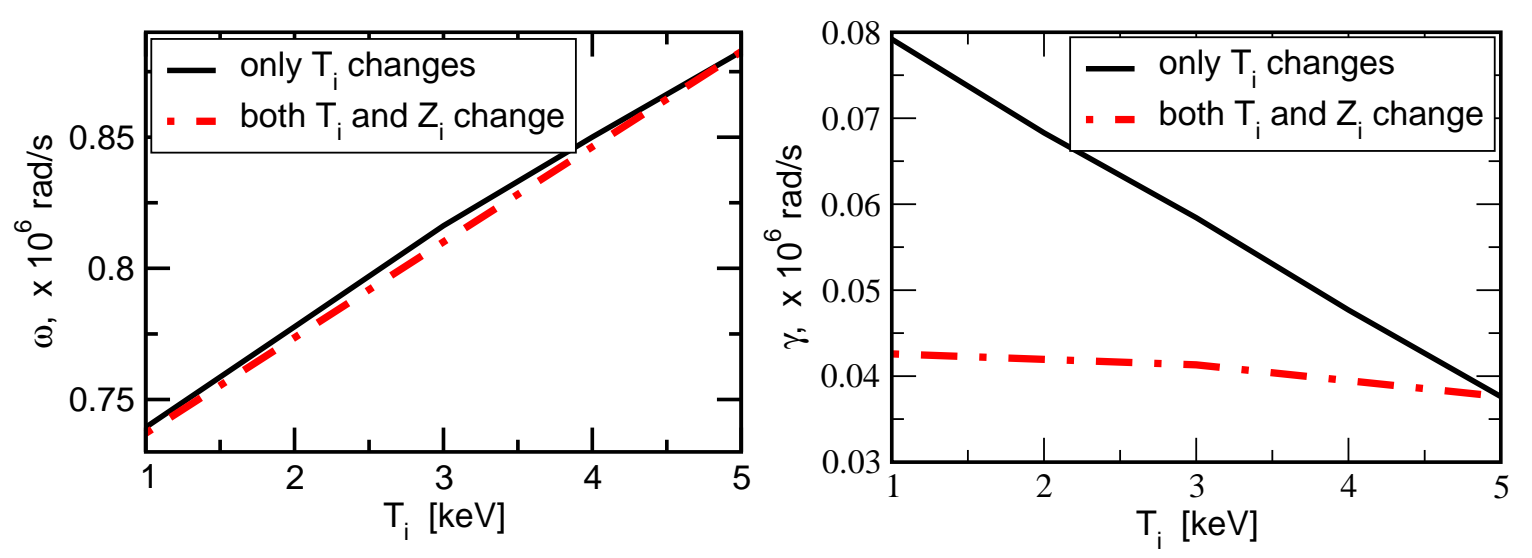

FIG. 15: (Color online): The frequency (on the left) and the growth rate (on the right) as a function of the bulk plasma temperature (flat profile). The solid line corresponds to the fixed bulk-ion charge number $Z_{i}=1$. For the dashed line, the bulk-ion charge number is adjusted with the bulk-ion temperature so that the bulk-ion gyroradius is constant (hence the radiative damping does not change). One sees that the growth rate remains almost constant for the dashed line signalising that the damping associated with the bulk-plasma FLR (i.e. the radiative damping) is the dominant mechanism for the case considered. 